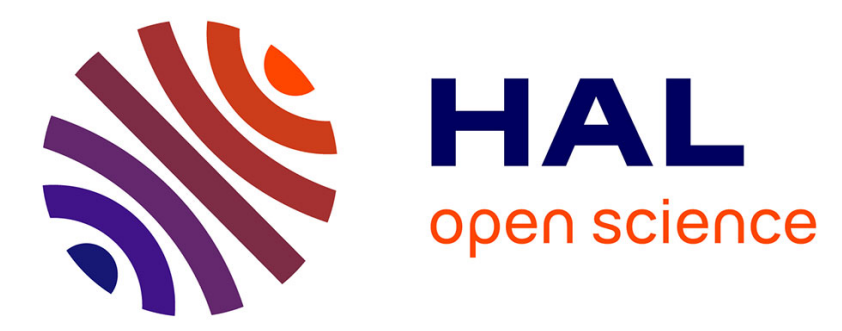

\title{
Emulation-based Dynamic Output-Feedback Control of Saturating Discrete-time LPV Systems
}

\author{
C. de Souza, V. J S Leite, Sophie Tarbouriech, E. B Castelan
}

\section{To cite this version:}

C. de Souza, V. J S Leite, Sophie Tarbouriech, E. B Castelan. Emulation-based Dynamic OutputFeedback Control of Saturating Discrete-time LPV Systems. IEEE Control Systems Letters, 2021, 5 (5), pp.2475-1456. 10.1109/LCSYS.2020.3040683 . hal-03069629

\section{HAL Id: hal-03069629 \\ https://hal.laas.fr/hal-03069629}

Submitted on 15 Dec 2020

HAL is a multi-disciplinary open access archive for the deposit and dissemination of scientific research documents, whether they are published or not. The documents may come from teaching and research institutions in France or abroad, or from public or private research centers.
L'archive ouverte pluridisciplinaire HAL, est destinée au dépôt et à la diffusion de documents scientifiques de niveau recherche, publiés ou non, émanant des établissements d'enseignement et de recherche français ou étrangers, des laboratoires publics ou privés. 


\title{
Emulation-based Dynamic Output-Feedback Control of Saturating Discrete-time LPV Systems
}

\author{
C. de Souza, V. J. S. Leite, S. Tarbouriech, and E. B. Castelan
}

\begin{abstract}
This paper addresses the problem of emulation-based dynamic output-feedback control for discrete-time linear parameter varying systems under actuator saturation. Sufficient conditions based on linear matrix inequalities (LMI) are derived to design the parameters of two dependent event-triggering rules handling the sensor-to-controller and controller-to-actuator communication and to guarantee the regional asymptotic stability of the closed-loop system. A convex optimization scheme incorporating these conditions is also formulated to reduce the output and control signals' update rate through communication channels. A numerical example illustrates the usefulness of the proposal.
\end{abstract}

Index Terms-Emulation-based control; Linear parameter-varying systems; Actuator saturation; Stability analysis.

\section{INTRODUCTION}

Recently, networked control systems (NCSs) have attracted significant attention due to their potential advantages comprising high reliability and low-cost installation and maintainability [1]. Although the network infrastructure brings us numerous benefits, some problems arise with its introduction, such as network congestion and elevated energy consumption on wireless systems. In this sense, it is meaningful to introduce a mechanism to reduce the unnecessary waste of communication resources and computation as in a periodic framework. As stated in [2], the so-called "event-triggering" scheme can reduce the number of data transmissions while guaranteeing a certain performance of the closed-loop system. Event-triggered control (ETC) is a strategy in which the data are transmitted only when a certain event (generated by a specified eventtriggering scheme) indicates an update is required. The existing approaches can be divided into two general categories, namely, emulation based [2]-[5] and co-design based [6], [7]. The co-design performs the design of both the controller and

C. de Souza (carla.souza93@hotmail.com) is with PPGEAS of Universidade Federal de Santa Catarina (UFSC), DAS/CTC/UFSC, 88040900, Florianópolis, SC, Brazil.

S. Tarbouriech (sophie.tarbouriech@laas.fr) is with LAAS-CNRS, Université de Toulouse, CNRS, Toulouse, France.

V. J. S. Leite (valter@ieee.org) is with the Department of Mechatronics Engineering, Campus Divinópolis - CEFET-MG, R. Álvares Azevedo, 400, 35503-822, Divinópolis, MG, Brazil. V. J. S. Leite is also with the the Graduate Program on Electrical Engineering (PPGEL) of CEFET-MG / UFSJ, Av. Amazonas 7675, Belo Horizonte, MG, Brazil.

E. B. Castelan (eugenio.castelan@ufsc.br) is of the Department of Automation and Systems, Universidade Federal de Santa Catarina (UFSC), PPGEAS - DAS/CTC/UFSC, 88040-900, Florianópolis, SC, Brazil.

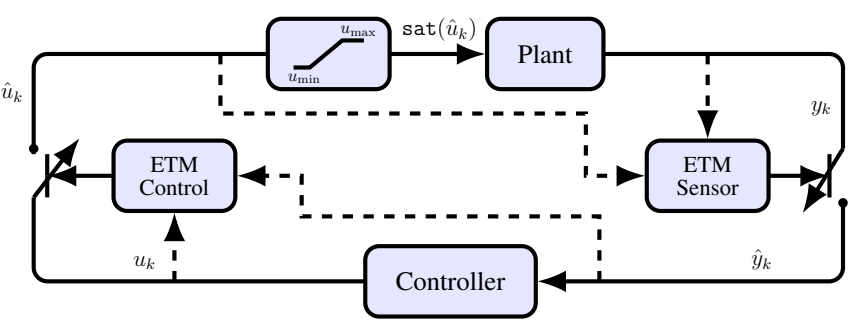

Fig. 1: Emulation-based control loop.

the event-triggering rule, simultaneously. On the other hand, the emulation considers that the controller is given a priori, and the goal is to design only the event-triggering rule.

In the current paper, we focus on the emulation design of event-triggered control for linear-parameter varying (LPV) systems with actuator saturation, as shown in Figure 1. In this case, a parameter-dependent dynamic output-feedback controller that stabilizes the system is previously computed without taking the event-triggering nature of the control system into account. Two dependent event-triggering mechanisms (ETM) are introduced in the loop to reduce the number of data transmissions between both sensor-to-controller and controller-to-actuator channels. Unlike the works in the literature, we consider that each ETM can use information from the other transmitted signal, which provides an enlarged set of information about the behavior of the system. As a consequence, the usage of the communication resources can be significantly saved.

Despite significant advancements in LPV systems analysis and control synthesis, only a few works on LPV eventtriggered control design are available in the literature. The event-based reference tracking control problem for discretetime LPV systems is addressed in [8], where a state-feedback controller and three event-triggering conditions are simultaneously designed. [9] investigates the event-triggered $\mathcal{H}_{\infty}$ control for discrete-time LPV systems by jointly designing a mixed ETM and a set of state feedback controllers. [10] addresses the problem of discretization and digital output feedback control design for continuous-time LPV systems subject to a time-varying networked-induced delay. The codesign problem of a state-feedback controller and an event generator is studied for discrete-time LPV systems in [11], where the scheduling variables are assumed to be not precisely known, but their estimates satisfy known uncertainty levels. However, the saturation is not considered in these works. 
Since the actuator saturation exists in almost every practical application, its effect on event-triggered control has been studied in recent publications. In [12], a local exponentially stability is guaranteed by a designed event-triggering condition concerning an LQ cost function. The dynamic event-triggered and self-triggered output feedback strategy is investigated in [13] for linear plants subject to input saturation and antiwindup compensation. [14] designs an output-based event generator and an anti-windup compensator for a linear system with actuator saturation. [15] proposes a methodology to design stabilizing event-triggering strategies for PI-controlled linear continuous-time plants subject to input saturation. The co-design problem of an event-triggered controller based on a static/dynamic feedback is investigated in [16] for stabilization of discrete-time systems subject to actuator saturation.

Given the previous discussions, the main contributions of this paper can be summarized as: i) An event-triggering strategy is proposed for discrete-time LPV systems with input saturation controlled by LPV dynamic output-feedback controllers; ii) Two ETMs are designed for transmitting the sensor measurements and the controller outputs through a communication network; iii) The proposed ETMs are dependent, i.e. each ETM can use information from the other transmitted signal; iv) The event-triggering rules are designed to ensure the asymptotic stability of the closed-loop system. An estimate of the origin's basin of attraction is characterized, and the data transmission activity is then indirectly reduced thanks to adequate optimization procedures. The convex conditions are based on a parameter-dependent Lyapunov function, which yields a linear matrix inequality (LMI) based formulation. A numerical example borrowed from the literature allows for depicting our approach's effectiveness compared with other literature methods.

Notation: $\mathbb{R}$ is the set of real numbers. 0 represents the null matrix of appropriate dimension and $\mathbf{I}_{n}$ corresponds to the identity matrix with dimensions $n \times n$. $\mathbb{R}^{m \times n}$ stands for the set of matrices with real entries of dimensions $m \times n$. A block-diagonal matrix $A$ with blocks $A_{1}$ and $A_{2}$ is denoted as $A=\operatorname{diag}\left\{A_{1}, A_{2}\right\}$. The $\ell^{\text {th }}$ row of the vector or matrix $A$ is indicated by $A_{(\ell)}$, and for a square matrix $A$ its diagonal element at $(\ell, \ell)$ is noted $A_{(\ell, \ell)}$. The set of integer numbers belonging to the interval from $a \in \mathbb{N}$ to $b \in \mathbb{N}, b \geq a$, is denoted by $\mathcal{I}[a, b]$. The symbol $\star$ represents the transpose blocks in relation to the diagonal of real square and symmetric matrices. For $x \in \mathbb{R}^{n},\|x\|$ is the Euclidean norm, and $\|x\|_{Q}^{2}$ is defined by $x^{\top} Q x$ with $0<Q=Q^{\top} \in \mathbb{R}^{n \times n}$.

\section{Problem Formulation}

Consider the saturating discrete-time LPV system represented by:

$$
\begin{aligned}
x_{p, k+1} & =A\left(\theta_{k}\right) x_{p, k}+B\left(\theta_{k}\right) \operatorname{sat}\left(\hat{u}_{k}\right), \\
y_{k} & =C x_{p, k},
\end{aligned}
$$

where $x_{p, k} \in \mathbb{R}^{n}$ is the state space vector, $y_{k} \in \mathbb{R}^{p}$ is the measured output, $\hat{u}_{k}$ is the updated control signal, and sat $\left(\hat{u}_{k}\right)$ is the standard decentralized saturation function, which is given by

$$
\operatorname{sat}\left(\hat{u}_{k(\ell)}\right)=\operatorname{sign}\left(\hat{u}_{k(\ell)}\right) \min \left(\left|\hat{u}_{k(\ell)}\right|, \bar{u}_{(\ell)}\right),
$$

with $\bar{u}_{(\ell)}>0, \ell \in \mathcal{I}[1, m]$, the symmetric magnitude bound relative to the $\ell^{\text {th }}$ update control input. The parameter-varying matrices $A\left(\theta_{k}\right) \in \mathbb{R}^{n \times n}$ and $B\left(\theta_{k}\right) \in \mathbb{R}^{n \times m}$ belong to a polytope

$$
(A, B)\left(\theta_{k}\right)=\sum_{i=1}^{N} \theta_{k(i)}\left(A_{i}, B_{i}\right), \forall \theta_{k} \in \Theta
$$

i.e., the vector of parameter-varying $\theta_{k} \in \mathbb{R}^{N}$ lies in the unit simplex given by

$$
\Theta \triangleq\left\{\sum_{i=1}^{N} \theta_{k(i)}=1, \theta_{k(i)} \geq 0, i \in \mathcal{I}[1, N]\right\} .
$$

As usual in emulation-based approach [17], we assume that the following parameter-dependent dynamic output-feedback (DOF) controller is given and stabilizes the system (1), as represented in Figure 1,

$$
\begin{aligned}
x_{c, k+1} & =A_{c}\left(\theta_{k}\right) x_{c, k}+B_{c}\left(\theta_{k}\right) \hat{y}_{k}-E_{c}\left(\theta_{k}\right) \Psi\left(\hat{u}_{k}\right), \\
u_{k} & =C_{c}\left(\theta_{k}\right) x_{c, k}+D_{c}\left(\theta_{k}\right) \hat{y}_{k},
\end{aligned}
$$

where $x_{c, k} \in \mathbb{R}^{n_{c}}$ is the control state, $\Psi\left(\hat{u}_{k}\right): \mathbb{R}^{m} \rightarrow \mathbb{R}^{m}$ is the dead-zone non-linearity defined by $\Psi\left(\hat{u}_{k}\right)=\hat{u}_{k}-\operatorname{sat}\left(\hat{u}_{k}\right)$, and $\hat{y}_{k}$ is the updated output signal. It is worth to say that the matrix $E_{c}\left(\theta_{k}\right)$ only mitigates the windup effects of the saturation and, therefore, can be set to zero in this approach if it has not been considered a priori. The controller matrices verify the following assumption:

Assumption 1: The parameter-varying matrices $A_{c}\left(\theta_{k}\right) \in$ $\mathbb{R}^{n_{c} \times n_{c}}, B_{c}\left(\theta_{k}\right) \in \mathbb{R}^{n_{c} \times p}, C_{c}\left(\theta_{k}\right) \in \mathbb{R}^{m \times n_{c}}, D_{c}\left(\theta_{k}\right) \in$ $\mathbb{R}^{m \times p}$, and $E_{c}\left(\theta_{k}\right) \in \mathbb{R}^{n \times m}$ of the DOF controller (5) have the following structure:

$$
\begin{aligned}
& \left(A_{c}, B_{c}\right)\left(\theta_{k}\right)=\sum_{i=1}^{N} \sum_{j=i}^{N}\left(1+\nu_{i j}\right) \theta_{k(i)} \theta_{k(j)}\left(\frac{A_{c i j}}{2}, \frac{B_{c i j}}{2}\right), \\
& \left(C_{c}, D_{c}\right)\left(\theta_{k}\right)=\sum_{i=1}^{N} \theta_{k(i)}\left(C_{c i}, D_{c i}\right), E_{c}\left(\theta_{k}\right)=\sum_{i=1}^{N} \theta_{k(i)} E_{c i},
\end{aligned}
$$

with $\theta_{k} \in \Theta$ and $\nu_{i j}=1$ if $i \neq j$ and $\nu_{i j}=0$ otherwise.

Note that, whenever the matrices of a dynamic controllers have a polytopic structure, they can always be cast in the structure given in Assumption 1.

To reduce the number of data exchange on sensor-tocontroller and controller-to-actuator channels, we are employing two dependent ETMs, as shown in Figure 1. In this case, the decision for output updates is made by the following rule:

$$
\hat{y}_{k}:= \begin{cases}y_{k}, & f(y, \hat{y}, \hat{u})>0, \\ \hat{y}_{k-1}, & \text { otherwise }\end{cases}
$$

where $f(y, \hat{y}, \hat{u})$ is the triggering condition defined by

$$
\left\|\hat{y}_{k-1}-y_{k}\right\|_{Q_{\Delta y}}^{2}-\left\|y_{k}\right\|_{Q_{y}}^{2}-\left\|\hat{u}_{k}\right\|_{Q_{\hat{u}}}^{2}>0,
$$

and the decision for the control updates is made by the following rule:

$$
\hat{u}_{k}:= \begin{cases}u_{k}, & g(u, \hat{u}, \hat{y})>0, \\ \hat{u}_{k-1}, & \text { otherwise },\end{cases}
$$


where $g(u, \hat{u}, \hat{y})$ is the triggering condition defined by

$$
\left\|\hat{u}_{k-1}-u_{k}\right\|_{Q_{\Delta u}}^{2}-\left\|u_{k}\right\|_{Q_{u}}^{2}-\left\|\hat{y}_{k}\right\|_{Q_{\hat{y}}}^{2}>0
$$

The positive definite matrices $Q_{\Delta y}, Q_{\hat{y}}, Q_{y} \in \mathbb{R}^{p \times p}$, and $Q_{\Delta u}, Q_{\hat{u}}, Q_{u} \in \mathbb{R}^{m \times m}$ are variables to be designed, and their choice directly affects the ETM performance. The interest of the structure of ETM (6)-(9) resides in the use of more information from the system behavior to decide to transmit or not the signals, expanding the event-triggering rules proposed in [22]. Therefore, the problem we intend to solve can be summarized as follows.

Problem 1: Consider the DOF controller (5) that regionally stabilizes the saturating LPV system (1) in the absence of a communication network. Design the two ETMs (6) and (8), such that the closed-loop asymptotic stability is preserved and the number of data transmissions on the sensor-to-controller and controller-to-actuator channels is as low as possible.

An implicit objective in solving Problem 1 is to characterize an estimate of the basin of attraction of the origin of the closedloop system [19].

\section{Preliminary Results}

Let us define the augmented state vector $x_{k}=$ $\left[\begin{array}{ll}x_{p, k}^{\top} & x_{c, k}^{\top}\end{array}\right]^{\top} \in \mathbb{R}^{n+n_{c}}$, the output error $e_{y, k}=\hat{y}_{k}-y_{k}$, and the control error $e_{u, k}=\hat{u}_{k}-u_{k}$. Thus, the closed-loop system can be written as

$$
\begin{aligned}
x_{k+1} & =\mathbb{A}\left(\theta_{k}\right) x_{k}-\mathbb{B}\left(\theta_{k}\right) \Psi\left(\hat{u}_{k}\right)+\mathbb{E}_{y}\left(\theta_{k}\right) e_{y, k}+\mathbb{E}_{u}\left(\theta_{k}\right) e_{u, k}, \\
u_{k} & =\mathbb{K}\left(\theta_{k}\right) x_{k}+D_{c}\left(\theta_{k}\right) e_{y, k}, \\
y_{k} & =\mathbb{C} x_{k} .
\end{aligned}
$$

where, as in Assumption 1, the closed-loop matrices verify

$$
\begin{aligned}
& \left(\mathbb{A}, \mathbb{E}_{y}\right)\left(\theta_{k}\right)=\sum_{i=1}^{N} \sum_{j=i}^{N}\left(1+\nu_{i j}\right) \theta_{k(i)} \theta_{k(j)}\left(\frac{\mathbb{A}_{i j}}{2}, \frac{\mathbb{E}_{y i j}}{2}\right), \\
& \left(\mathbb{B}, \mathbb{E}_{u}, \mathbb{K}^{\top}\right)\left(\theta_{k}\right)=\sum_{i=1}^{N} \theta_{k(i)}\left(\mathbb{B}_{i}, \mathbb{E}_{u i}, \mathbb{K}_{i}^{\top}\right),
\end{aligned}
$$

with $\theta_{k} \in \Theta, \nu_{i j}=1$ if $i \neq j$ and $\nu_{i j}=0$ otherwise, and are given by

$$
\begin{aligned}
\mathbb{A}_{i j} & =\left[\begin{array}{cc}
A_{i}+A_{j}+\left(B_{i} D_{c j}+B_{j} D_{c i}\right) C & B_{i} C_{c j}+B_{j} C_{c i} \\
B_{c i j} C & A_{c i j}
\end{array}\right], \\
\mathbb{B}_{i} & =\left[\begin{array}{c}
B_{i} \\
E_{c i}
\end{array}\right], \mathbb{E}_{u i}=\left[\begin{array}{c}
B_{i} \\
\mathbf{0}
\end{array}\right], \mathbb{E}_{y i j}=\left[\begin{array}{cc}
B_{i} D_{c j}+B_{j} D_{c i} \\
B_{c i j}
\end{array}\right], \\
\mathbb{K}_{i} & =\left[\begin{array}{ll}
D_{c i} C & C_{c i}
\end{array}\right], \text { and } \mathbb{C}=\left[\begin{array}{ll}
C & \mathbf{0}
\end{array}\right] .
\end{aligned}
$$

Note that if (6) activates an update at instant $k$, then from (7) we have that $e_{y, k}=\hat{y}_{k}-y_{k}=y_{k}-y_{k}=\mathbf{0}$. Otherwise, if $y_{k}$ is not updated at instant $k$, then from (6) we have that $e_{y, k}=\hat{y}_{k}-y_{k}=\hat{y}_{k-1}-y_{k}$. Thus, the following inequality is always satisfied.

$$
\left\|e_{y, k}\right\|_{Q_{\Delta y}}^{2} \leq\left\|y_{k}\right\|_{Q_{y}}^{2}+\left\|\hat{u}_{k}\right\|_{Q_{\hat{u}}}^{2} .
$$

Similarly, if (8) activates an update at instant $k$, then from (9) we have that $e_{u, k}=\hat{u}_{k}-u_{k}=\hat{u}_{k-1}-u_{k}=\mathbf{0}$. Otherwise, and if $u_{k}$ is not updated at instant $k$, then from (8) we have that $e_{u, k}=\hat{u}_{k}-u_{k}=\hat{u}_{k-1}-u_{k}$. Consequently, we always have

$$
\left\|e_{u, k}\right\|_{Q_{\Delta u}}^{2} \leq\left\|u_{k}\right\|_{Q_{u}}^{2}+\left\|\hat{y}_{k}\right\|_{Q_{\hat{y}}}^{2} .
$$

To investigate the emulation-based design, we use the Lyapunov theory with the following candidate Lyapunov function

$$
V\left(x_{k}\right)=x_{k}^{\top} P\left(\theta_{k}\right) x_{k},
$$

where $P\left(\theta_{k}\right)=\sum_{i=1}^{N} \theta_{k(i)} P_{i}$, with $0<P_{i}=P_{i}^{\top} \in$ $\mathbb{R}^{\left(n+n_{c}\right) \times\left(n+n_{c}\right)}, i \in \mathcal{I}[1, N]$, and $\theta_{k} \in \Theta$.

From the function $V\left(x_{k}\right)$ defined in (13), one can define the unitary level set $\mathcal{L}_{\mathcal{V}}=\left\{x_{k} \in \mathbb{R}^{n+n_{c}}: V\left(x_{k}\right) \leq 1, \forall \theta_{k} \in \Theta\right\}$. In Theorem 1 below, sufficient conditions are provided to ensure that the level set $\mathcal{L}_{\mathcal{V}}$ is an invariant and contractive set with respect to the trajectories of the closed-loop system (10), and, therefore, constitutes an estimate of the basin of attraction for the system [19]. Furthermore, by taking inspiration from [20], we can state the following lemma showing how to compute the set $\mathcal{L}_{\mathcal{V}}$ from matrices $P_{i}$.

Lemma 1: The level set $\mathcal{L}_{\mathcal{V}}$, associated with $V\left(x_{k}\right)$ defined in (13), is computed as

$$
\mathcal{L}_{\mathcal{V}}=\bigcap_{\forall \theta_{k} \in \Theta} \mathcal{E}\left(P\left(\theta_{k}\right)\right)=\bigcap_{\forall i \in \mathcal{I}[1, N]} \mathcal{E}\left(P_{i}\right),
$$

where $\mathcal{E}\left(P_{i}\right)$ denotes the ellipsoidal sets $\mathcal{E}\left(P_{i}\right)=$ $\left\{x_{k} \in \mathbb{R}^{n+n_{c}}: x_{k}^{\top} P_{i} x_{k} \leq 1, \forall i \in \mathcal{I}[1, N]\right\}$.

Proof: $\quad x \in \mathcal{L}_{\mathcal{V}}$ is equivalent to $\forall \theta_{k} \in \Theta, V\left(x_{k}\right)<1$, which is equivalent to $x \in \bigcap_{\forall \theta_{k} \in \Theta} \mathcal{E}\left(P\left(\theta_{k}\right)\right)$. In addition $\bigcap_{\forall \theta_{k} \in \Theta} \mathcal{E}\left(P\left(\theta_{k}\right)\right) \subset \bigcap_{\forall i \in \mathcal{I}[1, N]} \mathcal{E}\left(P_{i}\right)$. On the other hand, to prove that $\bigcap_{\forall i \in \mathcal{I}[1, N]} \mathcal{E}\left(P_{i}\right) \subset \bigcap_{\forall \theta_{k} \in \Theta} \mathcal{E}\left(P\left(\theta_{k}\right)\right)$, consider $x \in \bigcap_{\forall i \in \mathcal{I}[1, N]} \mathcal{E}\left(P_{i}\right)$, then $x^{\top} P_{i} x<1 \forall i \in \mathcal{I}[1, N]$. This implies that $x \in \mathcal{E}\left(P\left(\theta_{k}\right)\right), \forall \theta_{k} \in \Theta$ or $x \in \bigcap_{\forall \theta_{k} \in \Theta} \mathcal{E}\left(P\left(\theta_{k}\right)\right)$.

The dead-zone function $\Psi\left(\hat{u}_{k}\right)$ enjoys a generalized sector condition as in Lemma 1.6 page 43 of [19] or its equivalent formulation proposed in Equation (7.20) in Remark 7.4 in [19], as summarized in the lemma below.

Lemma 2: Consider a matrix $G\left(\theta_{k}\right)=\sum_{i=1}^{N} \theta_{k(i)} G_{i}$ with $G_{i} \in \mathbb{R}^{m \times n+n_{c}}$ for $\mathcal{I}[1, N], \theta_{k} \in \Theta$ and define the set

$$
\mathcal{S}(\bar{u}) \triangleq\left\{x_{k} \in \mathbb{R}^{n+n_{c}}:\left|G\left(\theta_{k}\right) x_{k}\right| \leq \bar{u}\right\} .
$$

If $x_{k} \in \mathcal{S}(\bar{u})$, then for any diagonal positive definite matrix $S \in \mathbb{R}^{m \times m}$, then the following inequality holds

$$
\Psi\left(\hat{u}_{k}\right)^{\top} S\left(G\left(\theta_{k}\right) x_{k}-\operatorname{sat}\left(\hat{u}_{k}\right)\right) \leq \mathbf{0} .
$$

\section{MAIN RESUlts}

The following theorem states a first step to solve Problem 1 .

Theorem 1: Suppose that there exist symmetric positive definite matrices $P_{i} \in \mathbb{R}^{\left(n+n_{c}\right) \times\left(n+n_{c}\right)}, Q_{\Delta u}, Q_{\hat{u}}, \hat{Q}_{u} \in$ $\mathbb{R}^{m \times m}$ and $Q_{\Delta y}, Q_{\hat{y}}, \hat{Q}_{y} \in \mathbb{R}^{p \times p}$, a positive definite diagonal matrix $S \in \mathbb{R}^{m \times m}$, and matrices $F \in \mathbb{R}^{\left(n+n_{c}\right) \times\left(n+n_{c}\right)}$ and $Z_{i} \in \mathbb{R}^{m \times\left(n+n_{c}\right)}$, with $i \in \mathcal{I}[1, N]$, such that (17) (given at the top of the next page) and

$\left[\begin{array}{cc}P_{i} & \star \\ Z_{i(\ell)} & 2 S_{(\ell, \ell)}-\bar{u}_{(\ell)}^{2}\end{array}\right]>\mathbf{0}, \quad\left\{\begin{array}{l}i \in \mathcal{I}[1, N] \\ \ell \in \mathcal{I}[1, m],\end{array}\right.$ 


\begin{tabular}{|c|c|c|c|c|c|}
\hline$-P\left(\theta_{k+1}\right)$ & $\star$ & $\star$ & $\star$ & $\star$ & \\
\hline (2) & $\begin{array}{c}P\left(\theta_{k}\right)-\mathbb{C}^{\top}\left(Q_{y}+Q_{\hat{y}}\right) \mathbb{C} \\
-\mathbb{K}\left(\theta_{k}\right)^{\top}\left(Q_{u}+Q_{\hat{u}}\right) \mathbb{K}\left(\theta_{k}\right)\end{array}$ & $\star$ & $\star$ & $\star$ & \\
\hline $\mathbf{0}$ & $-Q_{\hat{u}}^{\top} \mathbb{K}\left(\theta_{k}\right)$ & $Q_{\Delta u}-Q_{\hat{u}}$ & $\star$ & $\star$ & $+\mathbf{H e}(\phi \psi)>\mathbf{0}$ \\
\hline 0 & $\begin{array}{c}-D_{c}\left(\theta_{k}\right)^{\top}\left(Q_{u}+Q_{\hat{u}}\right) \mathbb{K}\left(\theta_{k}\right) \\
-Q_{\hat{y}}^{\top} \mathbb{C}\end{array}$ & $-D_{c}\left(\theta_{k}\right)^{\top} Q_{\hat{u}}$ & $\begin{array}{c}Q_{\Delta y}-Q_{\hat{y}} \\
-D_{c}\left(\theta_{k}\right)^{\top}\left(Q_{u}+Q_{\hat{u}}\right) D_{c}\left(\theta_{k}\right)\end{array}$ & $\star$ & \\
\hline $\mathbf{0}$ & $-S\left(\mathbb{K}\left(\theta_{k}\right)-G\left(\theta_{k}\right)\right)$ & $-S$ & $-S D_{c}\left(\theta_{k}\right)$ & $2 S$ & \\
\hline
\end{tabular}

where $\phi=\left[\begin{array}{lllll}F^{\top} & \mathbf{0} & \mathbf{0} & \mathbf{0} & \mathbf{0}\end{array}\right]^{\top}$ and $\psi=\left[\begin{array}{lllll}-\mathbf{I}_{n+n_{c}} & \mathbb{A}\left(\theta_{k}\right) & \mathbb{E}_{u}\left(\theta_{k}\right) & \mathbb{E}_{y}\left(\theta_{k}\right) & -\mathbb{B}\left(\theta_{k}\right)\end{array}\right]$

in the literature [16], [21], [23], but without taking into account possible parameter variations, i.e., with $\varphi_{k}=0$. This model can be cast as in (1) by computing the vertex matrices with the extreme values of $\varphi_{k}$. We assume that $\varphi_{k}$ is available and that the following dynamic output feedback controller has been designed with the conditions proposed in [18] (by disregarding delay and perturbation): $A_{c 11}=$ $\left[\begin{array}{ll}-0.0022 & -0.0003 \\ -0.1399 & -0.0052\end{array}\right], A_{c 12}=\left[\begin{array}{ll}-0.0022 & -0.0005 \\ -0.1229 & -0.0124\end{array}\right], A_{c 22}=$ $\left[\begin{array}{ll}-0.0038 & -0.0005 \\ -0.1068 & -0.0156\end{array}\right], B_{c 11}=\left[\begin{array}{cc}-105.5816 & -1.4670 \\ 126.5691 & -70.8165\end{array}\right]$, $B_{c 12}=\left[\begin{array}{cc}-105.7887 & -1.5065 \\ 103.3699 & -74.8924\end{array}\right], \quad E_{c 1}=\left[\begin{array}{c}0.1311 \\ 10.3767\end{array}\right]$, $B_{c 22}=\left[\begin{array}{cc}-106.1570 & -1.5502 \\ 80.1418 & -78.6531\end{array}\right], E_{c 2}=\left[\begin{array}{l}0.1152 \\ 7.8952\end{array}\right], C_{c 1}=$ $\left[\begin{array}{ll}-0.0067 & -0.0003\end{array}\right], C_{c 2}=\left[\begin{array}{ll}-0.0067 & -0.0010\end{array}\right], D_{c 1}=$ $\left[\begin{array}{ll}7.7120 & 1.3814\end{array}\right]$, and $D_{c 2}=\left[\begin{array}{ll}7.7053 & 1.3193\end{array}\right]$, which ensures the regional asymptotic stability of the LPV system (22) in the absence of a communication network.

Problem 1 is addressed by considering both the LPV and linear time-invariant (LTI) cases. Then, by using the optimization procedure (21) with $\eta=19$, we got the following ETM matrices $Q_{\Delta y}=\left[\begin{array}{cc}18.3651 & 3.1401 \\ 3.1401 & 0.6349\end{array}\right], Q_{\hat{y}}=\left[\begin{array}{cc}0.1255 & -0.0239 \\ -0.0239 & 0.0045\end{array}\right]$, $Q_{y}=\left[\begin{array}{cc}8.9332 & -0.9033 \\ -0.9033 & 0.0913\end{array}\right], Q_{\Delta u}=19$, and $Q_{\hat{u}} \approx Q_{u} \approx$ $5 \times 10^{-11}$. The efficiency of the event-triggering strategy was assessed by simulating 1000 initial conditions generated with uniformly distributed random $x_{1}$ and $x_{2}$ in the intervals contained in $\mathcal{L}_{\mathcal{V}}: x_{1} \in \mathcal{I}[-0.04,0.04]$ and $x_{2} \in \mathcal{I}[-0.3,0.3]$, and the parameter-varying $\varphi_{k}=|\sin (0.01 k \pi)|$. The average rate of sensor updates and of control updates were $12 \%$ and $18.67 \%$, respectively. Also, by considering the initial conditions $x_{p, 0}=\left[\begin{array}{ll}0.0023 & 0.6639\end{array}\right]^{\top}$ and $x_{c, 0}=\left[\begin{array}{ll}0 & 0\end{array}\right]^{\top}$, which is in the $\mathcal{L}_{\mathcal{V}}$ border, we simulated the closed-loop system response, and plotted the states, the control input, the inter-events on the sensor and the inter-events on the controller as shown in Figure 2. We can see the regional asymptotic stability of the system with an output update rate of $13.67 \%$ and a control update rate of $21.33 \%$. Therefore, using an ETC strategy, we were able to significantly reduce the number of communications between process and controller, and controller and actuator.

Assuming time-invariant and know parameters $\left(\varphi_{k}=0\right)$, the inverted pendulum system is also investigated in [16], where both the static and dynamic feedback controllers under
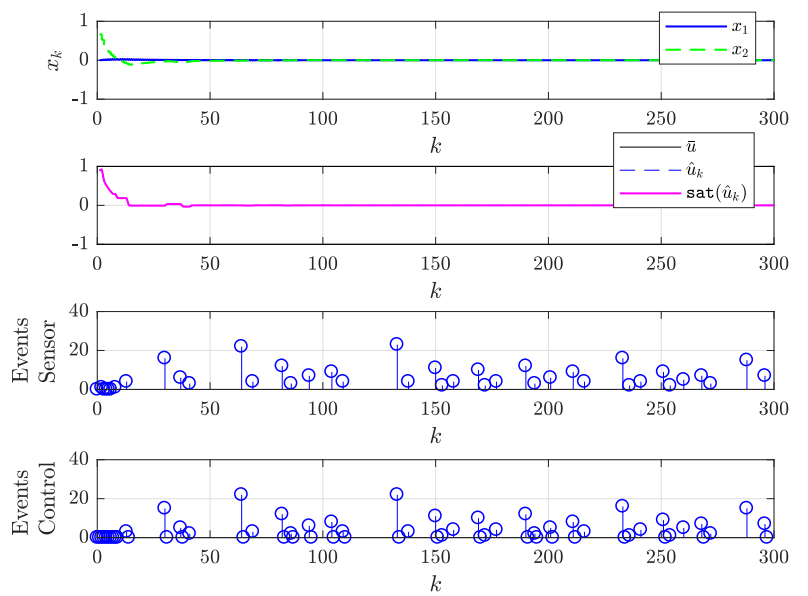

Fig. 2: The closed-loop response of system (22) for $x_{p, 0}=$ $\left[\begin{array}{ll}0.0023 & 0.6639\end{array}\right]^{\top}$ and $x_{c, 0}=\left[\begin{array}{ll}0 & 0\end{array}\right]^{\top}$.

an event-triggering mechanism are designed. For the dynamic controller, [16] obtained a sensor update rate of $6.7 \%$ in the simulation of the closed-loop response considering the initial condition $x_{p, 0}=\left[\begin{array}{ll}0.2 & 0.8\end{array}\right]^{\top}$ and $x_{c, 0}=\left[\begin{array}{ll}0 & 0\end{array}\right]^{\top}$. The control update rate in this case is the worst possible, $100 \%$, since [16] does not consider the presence of a communication network on the controller-to-actuator channel. The controller proposed by [16] is given by: $A_{c}=\left[\begin{array}{cc}0.8711 & -0.0489 \\ -2.3439 & 0.1301\end{array}\right]$, $B_{c}=\left[\begin{array}{cc}-0.0178 & -0.0312 \\ 0.0475 & 0.0821\end{array}\right], E_{c}=\left[\begin{array}{ll}0.0350 & -0.0589\end{array}\right]^{\top}$, $C_{c}=\left[\begin{array}{ll}-6.8922 & -1.7913\end{array}\right], D_{c}=\left[\begin{array}{ll}0.1934 & 1.0029\end{array}\right]$, that stabilizes the LTI system (22) in the absence of a communication network. To establish a fair comparison, we impose a region of admissible initial conditions with, at least, the same size of that achieved in [16] by ensuring $\mathcal{E}(R) \subseteq \mathcal{E}(P)$ with $R_{n}=\left[\begin{array}{cc}37.3546 & -4.7078 \\ -4.7078 & 1.5614\end{array}\right]$ (defined in the optimization procedure section). Then, by using the optimization procedure (21) with $\eta=1$, we got the following ETM matrices $Q_{\Delta y}=\left[\begin{array}{ll}0.1212 & 0.2381 \\ 0.2381 & 0.8788\end{array}\right], Q_{\hat{y}}=\left[\begin{array}{ll}0.0348 & 0.0109 \\ 0.0109 & 0.0168\end{array}\right], Q_{y}=$ $\left[\begin{array}{ll}0.1398 & 0.0163 \\ 0.0163 & 0.0019\end{array}\right], Q_{\Delta u}=1$, and $Q_{\hat{u}} \approx Q_{u} \approx 2 \times 10^{-10}$. Figure 3 depicts the projection (-), the cut $(-)$ of $\mathcal{L}_{\mathcal{V}}$, and $\mathcal{E}(R)(-)$. Note that $\mathcal{E}(R)$ is a subset of $\mathcal{L}_{\mathcal{V}}$ as required. Also, the projection of some divergent (-) and some convergent (- 
) trajectories stating from the points marked with $(+)$ and $(*)$, respectively, are illustrated. By considering the initial condition $x_{p, 0}=\left[\begin{array}{ll}0.2 & 0.8\end{array}\right]^{\top}$, used in [16], one obtains a closed-loop convergent trajectory (dashed black line) with a sensor update rate of $6.6 \%$ and a control update rate of $18.20 \%$. Although [16] has achieved a sensor update rate $1.52 \%$ smaller than ours, its control update rate is significantly higher than ours, in the order of $449.45 \%$.

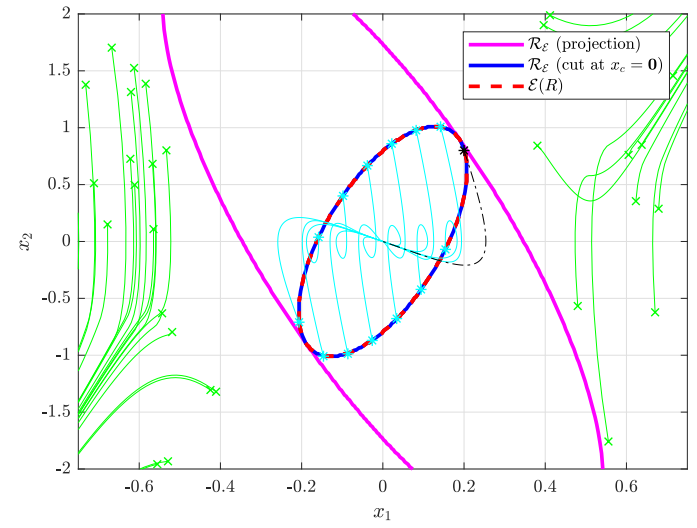

Fig. 3: The estimate of the basin of attraction $\mathcal{L}_{\mathcal{V}}$, projection $(-)$ and cut $(-)$, and the region of admissible initial conditions $\mathcal{E}(R),(-)$, found by [16].

\section{CONCLUSION}

This paper handled the problem of emulation-based dynamic output-feedback control for discrete-time linear parameter varying systems under actuator saturation. Two eventtriggering mechanisms were proposed to deal with the data transmission on the sensor-to-controller and controller-toactuator channels, while preserving the closed-loop stability. Sufficient conditions based on linear matrix inequalities (LMI) allowed to design the parameters of the two dependent eventtriggering rules and to characterize an estimate of the basin of attraction of the origin. A convex optimization scheme incorporating these conditions as constraints was proposed to reduce the number of updates on the sensor-to-controller and the controller-to-sensor channels. The effectiveness of the method has been tested in an illustrative example borrowed from the literature. Future work could be dedicated to expanding the results to the co-design problem, for example, by taking inspiration from preliminary results in [24].

\section{ACKNOWLEDGMENT}

This work has been supported by the Brazilian Agencies CAPES under the project Print CAPES-UFSC "Automation 4.0" and CNPq (Grants 311208/2019-3 and 306927/2017-9); and by ANR, project HANDY 18-CE40-0010.

\section{REFERENCES}

[1] J. P. Hespanha, P. Naghshtabrizi, and Y. Xu, "A survey of recent results in networked control systems," Proceedings of the IEEE, vol. 95, no. 1, pp. 138-162, 2007.

[2] W. Heemels, M. Donkers, and A. Teel, "Periodic event-triggered control for linear systems," IEEE Transactions on Automatic Control, vol. 58, no. 4, pp. 847-861, 2012.
[3] P. Tabuada, "Event-triggered real-time scheduling of stabilizing control tasks," IEEE Transactions on Automatic Control, vol. 52, no. 9, pp. 1680-1685, 2007.

[4] W. Heemels, K. H. Johansson, and P. Tabuada, "An introduction to event-triggered and self-triggered control," in 2012 IEEE 51st IEEE Conference on Decision and Control (CDC), 2012, pp. 3270-3285.

[5] W. Heemels and M. Donkers, "Model-based periodic event-triggered control for linear systems," Automatica, vol. 49, no. 3, pp. 698-711, 2013.

[6] C. Peng and T. C. Yang, "Event-triggered communication and $\mathcal{H}_{\infty}$ control co-design for networked control systems," Automatica, vol. 49, no. 5, pp. 1326-1332, 2013.

[7] M. Abdelrahim, R. Postoyan, J. Daafouz, and D. Nešić, "Co-design of output feedback laws and event-triggering conditions for linear systems," in 53rd IEEE Conference on Decision and Control. IEEE, 2014, pp. $3560-3565$.

[8] A. Golabi, N. Meskin, R. Tóth, J. Mohammadpour, T. Donkers, and M. Davoodi, "Event-triggered constant reference tracking control for discrete-time LPV systems with application to a laboratory tank system," IET Control Theory \& Applications, vol. 11, no. 16, pp. 2680-2687, 2017.

[9] S. Li, D. Sauter, and B. Xu, "Co-design of event-triggered $\mathcal{H}_{\infty}$ control for discrete-time linear parameter-varying systems with network-induced delays," Journal of the Franklin Institute, vol. 352, no. 5, pp. 1867-1892, 2015.

[10] M. F. Braga, C. F. Morais, E. S. Tognetti, R. C. L. F. Oliveira, and P. L. D. Peres, "Discretization and event triggered digital output feedback control of LPV systems," Systems \& Control Letters, vol. 86, pp. 54-65, 2015.

[11] S. Li and B. Xu, "Event-triggered control for discrete-time uncertain linear parameter-varying systems," in Proceedings of the 32nd Chinese Control Conference. IEEE, 2013, pp. 273-278.

[12] A. Seuret, C. Prieur, S. Tarbouriech, and L. Zaccarian, "Event-triggered control with LQ optimality guarantees for saturated linear systems," IFAC Proceedings Volumes, vol. 46, no. 23, pp. 341-346, 2013.

[13] Z. Zuo, S. Guan, Y. Wang, and H. Li, "Dynamic event-triggered and selftriggered control for saturated systems with anti-windup compensation," Journal of the Franklin Institute, vol. 354, no. 17, pp. 7624-7642, 2017.

[14] Z. Quan and D. Ma, "Output-based event-triggered control for linear systems with actuator saturation," in 2016 Chinese Control and Decision Conference (CCDC). IEEE, 2016, pp. 1500-1505.

[15] L. G. Moreira, L. B. Groff, J. Gomes da Silva Jr, and S. Tarbouriech, "PI event-triggered control under saturating actuators," International Journal of Control, vol. 92, no. 7, pp. 1634-1644, 2019.

[16] S. Ding, X. Xie, and Y. Liu, "Event-triggered static/dynamic feedback control for discrete-time linear systems," Information Sciences, 2020.

[17] J. Lunze and D. Lehmann, "A state-feedback approach to event-based control," Automatica, vol. 46, no. 1, pp. 211-215, 2010.

[18] C. de Souza, E. B. Castelan, and V. J. S. Leite, "Input-to-state stabilization of discrete-time LPV systems with bounded time-varying state delay and saturating actuators through a dynamic controller," in 2019 IEEE 58th Conference on Decision and Control (CDC). IEEE, 2019, pp. 3782-3787.

[19] S. Tarbouriech, G. Garcia, J. M. Gomes da Silva Jr., and I. Queinnec, Stability And Stabilization Of Linear Systems With Saturating Actuators. (Springer, 2011.

[20] M. Jungers and E. B. Castelan, "Gain-scheduled output control design for a class of discrete-time nonlinear systems with saturating actuators," Systems \& Control Letters, vol. 60, no. 3, pp. 169-173, 2011.

[21] L. Groff, L. Moreira, and J. Gomes da Silva, "Event-triggered control codesign for discrete-time systems subject to actuator saturation," in 2016 IEEE Conference on Computer Aided Control System Design (CACSD). IEEE, 2016, pp. 1452-1457.

[22] C. de Souza, S. Tarbouriech, E. B. Castelan, and V. J. S. Leite, "Event-triggered dynamic output-feedback controller for discrete-time LPV systems with constraints," in 24th International Symposium on Mathematical Theory of Networks and Systems, 2020, pp. 1-6, accepted.

[23] W. Wu, S. Reimann, and S. Liu, "Event-triggered control for linear systems subject to actuator saturation," IFAC Proceedings Volumes, vol. 47, no. 3, pp. 9492-9497, 2014.

[24] C. de Souza, V. J. S. Leite, S. Tarbouriech, and E. B. Castelan, "Eventtriggered policy for dynamic output stabilization of discrete-time LPV systems under input constraints," Systems \& Control Letters, 2020, Submitted. 